\title{
Saturated-fat diet induces moderate diabetes and severe glomerulosclerosis in hamsters
}

\author{
D. Popov ${ }^{1}$, M. Simionescu ${ }^{1}$, P. R. Shepherd ${ }^{2}$ \\ ${ }^{1}$ Institute of Cellular Biology and Pathology "N.Simionescu", Bucharest, Romania \\ ${ }^{2}$ Department of Biochemistry and Molecular Biology, University College of London, UK
}

\begin{abstract}
Aims/hypothesis. Diets high in saturated fat are thought to be a risk factor for Type 2 diabetes and associated complications. We investigated effects of a medium and high saturated fat diet on the development of diabetes-associated pathologies in Golden Syrian hamsters, an animal that reacts to dietary lipids in a fashion similar to humans.

Methods. Three diets containing $46.5 \mathrm{kcal} \%$, $267.3 \mathrm{kcal} \%$, and $488.2 \mathrm{kcal} \%$ as saturated fat respectively, were studied. Metabolic parameters were measured up to 20 weeks. Electron microscopy was used to examine the structure of the pancreas, aorta and kidney. Results. Increased saturated fat consumption was associated with: (i) gradual imbalance of homeostasis, and severe structural alterations of acinar, beta cells and capillaries in the pancreas, and of the kidney glomeruli; (ii) severe hypertriglyceridaemia and aug-
\end{abstract}

mented creatinine concentrations related to disturbances of the renal function, progressing to nodular glomerulosclerosis and nephropathy; (iii) reduced early insulin secretion in response to glucose; (iv) switch of the aortic endothelium to a secretory phenotype.

Conclusion/interpretation. The results show that highcaloric saturated fat intake induced diabetes in hamsters, probably linked to delayed insulin secretion. The model was also associated with the development of a range of pathologies characteristic to human diabetes, including nephropathy and defects in vasculature. Thus, high-fat fed hamsters provide a new model that is likely to be useful in understanding the cellular and molecular mechanisms involved in the pathogenesis of diabetes. [Diabetologia (2003) 46:1408-1418]

Keywords High-fat diet, Type 2 diabetes, beta cells, insulin secteron, glucose intolerance, nodular glomerulosclerosis, hypertriglyceridaemia.
The cause and effect interaction between high-fat diets and Type 2 diabetes is still a matter of debate. There are data linking high-fat diets to the development of diabetes $[1,2,3]$ but most studies use rat and mouse

Received: 11 February 2003 / Revised: 2 June 2003

Published online: 29 July 2003

(C) Springer-Verlag 2003

Corresponding author: Dr. D. Popov, Institute of Cellular Biology and Pathology "N.Simionescu", 8, BP Hasdeu Street, Bucharest, 79691, Romania

E-mail: popov@simionescu.instcellbiopath.ro

Tel.: +40-1-4110860

Fax: +40-1-4111143

Abbreviations: GBM, Glomerular basement membrane. models $[4,5,6,7,8,9,10,11]$ whereby animals fed high-fat diets developed peripheral insulin resistance. Other animals used for studies of high-fat diets included dogs [12], rabbits [13], and hamsters [14, 15]. However, there has been a large variation in the protocols used to achieve these effects, in terms of the fatty-acid composition in the diet and its duration $[5,7$, $8,9,10,11,15,16,17]$.

Type 2 diabetic patients have an increased risk of developing atherosclerosis $[18,19,20,21]$. However, it has proven difficult to produce animal models that can be used to study the links between Type 2 diabetes and atherosclerosis. Mice and rats are resistant to developing atherosclerosis. However, we and others have reported that Golden Syrian hamsters can be in- 
duced to develop atherosclerosis in a manner that closely mimics human pathology. These hamsters have: (i) the lipid metabolism similar to humans [22, 23], (ii) the propensity to develop atherosclerosis in response to a diet supplemented with $3 \%$ cholesterol and $15 \%$ butter $[24,25]$.

To our knowledge, links between the development of diabetes and high-fat feeding in hamsters have only been investigated in one previous study [15], in which animals were fed for a maximum of 21 days with a diet containing around $25 \%$ fat (half unsaturated) with added cholesterol and 10\% fructose in the drinking water. The authors concluded that this animal is a suitable model for studying links between diabetes and dyslipidaemia.

We have used Golden Syrian hamsters maintained on diets enriched in saturated fat, but notably, we did not include extra cholesterol as we did in previous studies $[25,26]$.

The effects on the hamsters fed three diets containing different concentrations of saturated fat for periods of up to 20 weeks were compared for effects on: (i) homeostasis, (ii) glucose and insulin tolerance, (iii) the structure of the pancreatic beta cells, the aortic arch (as a lesion prone area for atherosclerosis), and the kidney glomerular capillaries (as targets of diabetic complications).

\section{Materials and methods}

Reagents. The enzymatic kits used for the assay of cholesterol, glucose, creatinine and triglycerides were obtained from Sigma (Mo., USA). Insulin was assayed with rat insulin ELISA kit purchased from DRG Instruments, Germany. Medisense (Bedford, Mass., USA) produced the Precision Q-I-D glucometer used in glucose-tolerance tests.

Animals. A total of 90 male one-year-old Golden Syrian hamsters weighing 130 to $145 \mathrm{~g}$ were used. The animals had free access to food and water, were housed in plastic cages, and were kept on a 12-h light-to-dark cycle. All experiments were carried out under the "Principles of laboratory animal care" (NIH publication no. 85-23, revised 1985).

Development of the hypercaloric-hamster model. Normal hamsters ( $n=30$, group C) were given standard pelleted Golden Syrian hamster diet [27]. Two groups of hamsters (30 animals each) received hypercaloric diets by supplementing the pelleted chow with lard in two doses: $2.5 \mathrm{~g}$ lard+7.5 g chow ( $267.3 \mathrm{kcal} \%$ as fat, corresponding to $28.74 \%$ fat in the diet) and $5 \mathrm{~g} \mathrm{lard}+5 \mathrm{~g}$ chow $(488.2 \mathrm{kcal} \%$ as fat, i.e. $52.49 \%$ fat in the diet). The energy concentration of the diets used was: $1.94 \mathrm{MJ} / \mathrm{kg}$ in group C, and 11.18 and $20.42 \mathrm{MJ} / \mathrm{kg}$. As indicated animals were killed and the weight of the epididymal fat and of the retroperitoneal fat was measured. The amount of visceral fat was calculated and adiposity index was calculated by dividing the sum of the visceral fat by (body weight-visceral fat weight) $\times 100$.

Biochemical assays. At the start of the experiment, and then after 2, 4, 10 and 20 weeks, hamsters fed control and hyperca- loric diets were lightly anesthetized by i.p. injection of $5 \%$ chloralhydrate $(0.05 \mathrm{ml} / 100 \mathrm{~g}$ b.w.). Blood was collected from the venous retroorbital plexus. Plasma (blood collected on $2.7 \mathrm{mmol} / \mathrm{l}$ EDTA) was used for the measurement of cholesterol, glucose and triglyceride concentrations while the creatinine was assayed in serum.

Glucose and insulin tolerance tests. For assessment of glucose tolerance, hamsters were fasted overnight, then saline solution containing $1 \mathrm{mg}$ glucose/g body weight was i.p. injected. Blood was withdrawn from the retroorbital plexus at $0,30,120$ and $180 \mathrm{~min}$, and blood glucose concentrations measured with the glucometer.

Plasma insulin concentrations were measured after fast, and at different time intervals after glucose (as above) and insulin injection ( $0.05 \mathrm{U} / 100 \mathrm{~g}$ body weight), at time intervals: 0,10 , 30 and 120 min using insulin ELISA kit.

HOMA index. The homeostatic model assessment (HOMA) is based on the concentration of basal fasting glucose ( $\mathrm{g}$ expressed in moles) and insulin (i, expressed in $\mu \mathrm{U} / \mathrm{ml}$ ) as described $[28,29]$. The insulin resistance is given by $\mathrm{R}_{\mathrm{HOMA}}=$ $\mathrm{g} \times \mathrm{i} / 22.5$ [30].

Tissue preparation for electron microscopy. Hamsters in either control or high-fat groups were anesthetized by an i.p. injections of $5 \%$ chloral hydrate $(0.1 \mathrm{ml} / 100 \mathrm{~g}$ body weight $)$. After laparotomy and catheterization of the abdominal aorta, the vena cava caudalis was punctured, and the blood was washed out by perfusion of phosphate buffered saline under a flow rate of $6 \mathrm{ml} / \mathrm{min}$. Under the same conditions, the tissues were fixed by perfusion of a mixture of $2.5 \%$ paraformaldehyde and $1.5 \%$ glutaraldehyde in $0.1 \mathrm{~mol} / 1 \mathrm{Na}$ cacodylate- $\mathrm{HCl}$ buffer, supplemented with $2.5 \mathrm{mmol} / \mathrm{l} \mathrm{CaCl}_{2}, \mathrm{pH}$ 7.4. After $10 \mathrm{~min}$, small fragments of the aortic arch, pancreas and kidney cortex were removed, kept for an additional $90 \mathrm{~min}$ in fixative, postfixed in $1 \% \mathrm{OsO}_{4}$, dehydrated in graded concentrations of ethanol, and embedded in Epon 812. Thin sections stained with $7.6 \%$ uranyl acetate (in distilled water) and $0.4 \%$ lead citrate (in $0.1 \mathrm{~N}$ $\mathrm{NaOH}$ ) were examined with the Phillips electron microscope (Eindhoven, The Netherlands).

Morphometric analysis. To quantitate the thickness of the glomerular basement membrane a previously described method [31] was used on electron micrographs of similar magnification $(\times 55,000)$.

Statistical analysis. All data were expressed as means \pm SEM. The differences among the three diet groups were analysed by ANOVA. Differences were considered significant at a $p$ value of less than 0.05 .

\section{Results}

Effects of high-fat diet on homeostasis. Control hamsters fed for 20 weeks with normal chow (46.5 kcal \% as fat) had normal amounts of food intake (i.e. an average of $10 \mathrm{~g} /$ day), no mortality or significant modification of body weight, and the calculated adiposity index was 2.9\%; the animals maintained on this diet had normal concentrations of circulating cholesterol, glucose, creatinine, triglycerides, and insulin throughout the 20 weeks of the experiment (Table 1). 
Table 1. General attributes of the Golden Syrian hamsters fed standard and hypercaloric diets

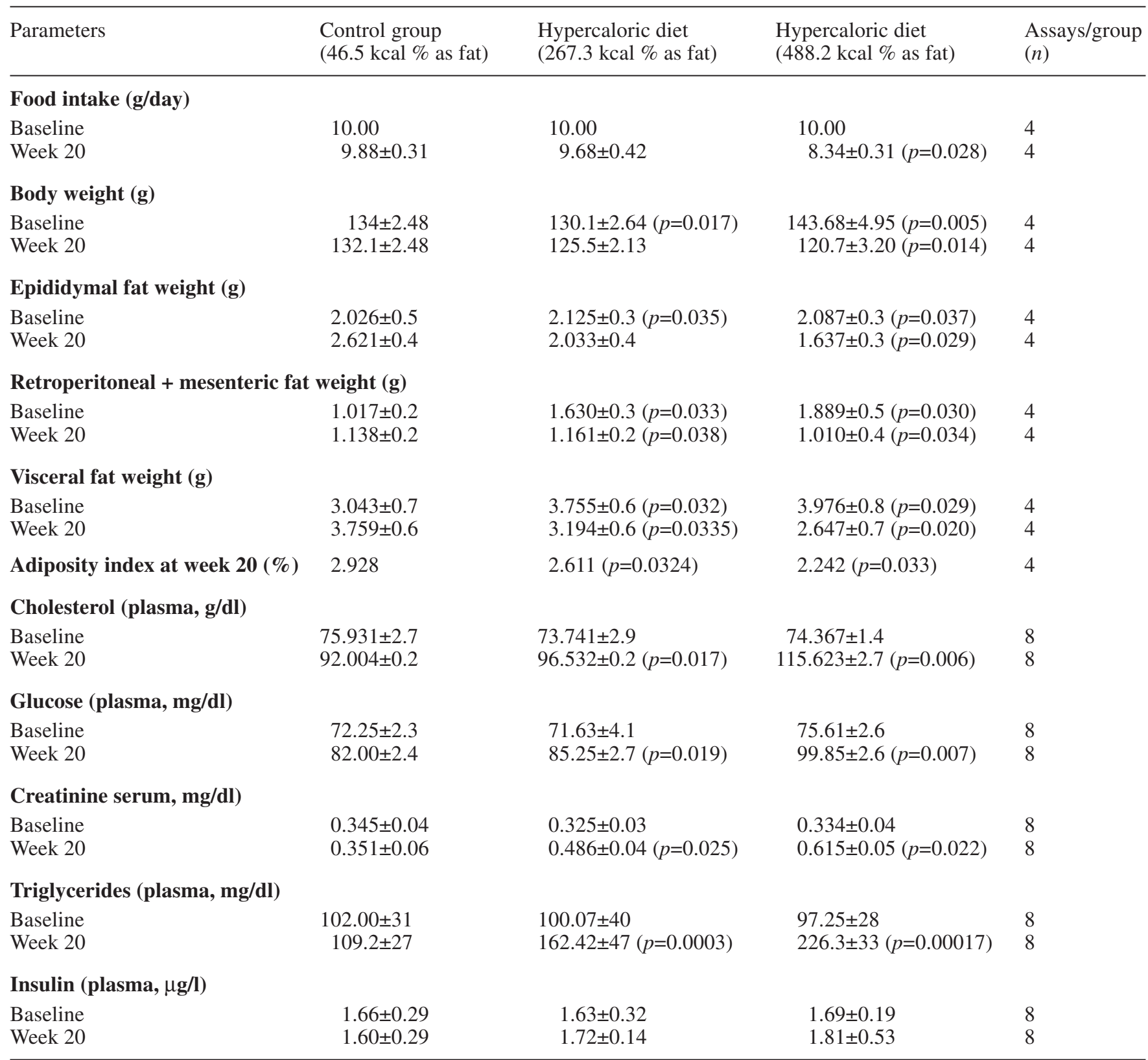

Values are means \pm SEM

Compared with the control animals, hamsters fed the diet containing $267.3 \mathrm{kcal} \%$ as fat, showed at 20 weeks a slightly diminished food intake $(\sim 2 \%)$ and body weight $(\sim 5 \%)$, decreased amount of visceral fat $(15 \%)$ and a reduced adiposity index $(11 \%)$. As compared to normal values, this group had a slightly augmented concentration of plasma cholesterol $(\sim 5 \%)$ and glucose $(\sim 4 \%)$ and a significant increase in creatinine $(\sim 38.4 \%)$ and triglycerides $(\sim 48.7 \%)$ (Table 1$)$.

The hamsters that received a total of $488.2 \mathrm{kcal} \%$ of fat for 20 weeks showed (compared to controls) a $15.6 \%$ decrease in food intake, approximately a $8.6 \%$ decrease in body weight, reduced amount of visceral fat and a diminished adiposity index (by 30\% and
$23.4 \%$, respectively). In this group, the concentration of plasma cholesterol increased by $25.6 \%$, plasma glucose by $22 \%$, creatinine by $75 \%$, and triglycerides by $207 \%$ above the control values (Table 1).

There was no change in the insulin concentration in the groups of animals fed normal or hypercaloric diets (Table 1).

Effects of high-fat diets on glucose disposal. The hamsters fed the two high-fat diets showed an impaired glucose tolerance (Fig. 1). Not only did the severity of the glucose intolerance increase with increasing concentrations of dietary fat (Fig. 1A) but it also became progressively worse with time on the diet (Fig. 1B). In 

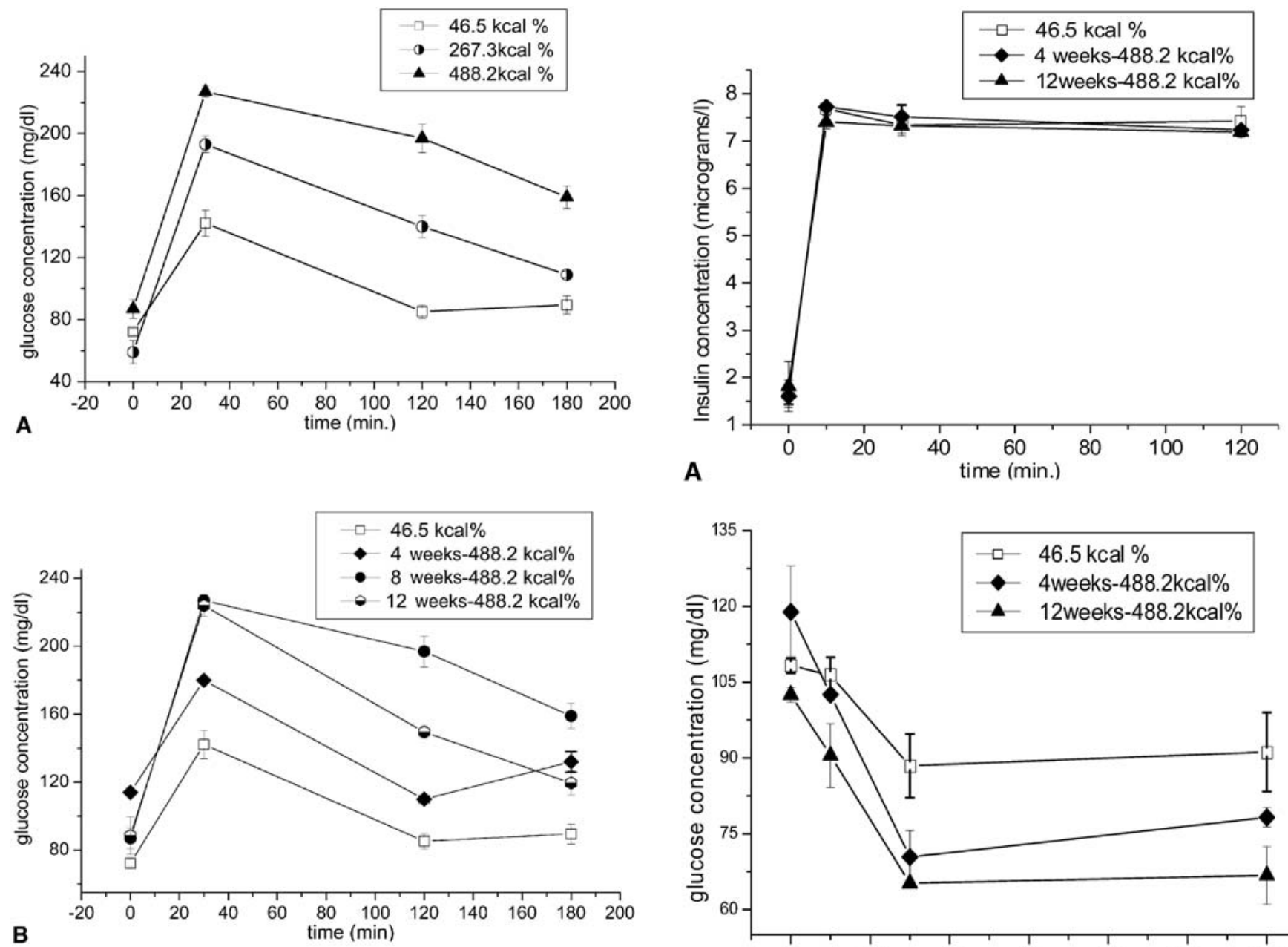

Fig. 1A, B. Effects of high-fat diet on glucose tolerance. Hamsters were fasted for $18 \mathrm{~h}$ prior to glucose tolerance tests (performed as described in methods). (A) Glucose tolerance at feeding hypercaloric (267.3 and $488.2 \mathrm{kcal} \%$ as fat) and control diet $(46.5 \mathrm{kcal} \%$ as fat) for 8 weeks. At $180 \mathrm{~min}$ time point both hypercaloric diets induced higher circulating glucose concentrations $(p=0.041$ at $267.3 \mathrm{kcal} \%$, and $p=0.0012$ at $488.2 \mathrm{kcal} \%$ ). (B) The glucose tolerance in hamsters fed $488.2 \mathrm{kcal} \%$ as fat for 4, 8 and 12 weeks. All groups of animals on hypercaloric diets showed increased circulating glucose concentrations ( $p<0.05$ vs controls) at $180 \mathrm{~min}$ after the glucose load. Each point on the diagrams represents experimental values data from five hamsters

an additional experiment we tested female hamsters under the same high-fat diets (as with the males group), and found that the effects of injected glucose were very similar for both sexes.

Insulin tolerance tests were carried out to determine whether the impaired glucose tolerance was due to insulin resistance in target tissues. Injected insulin (0.05 U/100 g b.w.) reached the circulation to an equal extent in both control and high-fat fed animals (Fig. 2A). Insulin reduced blood glucose more rapidly in the high-fat fed animals compared to controls (Fig. 2B). The results of the insulin tolerance test indicate that the high-fat fed animals are able to shutoff

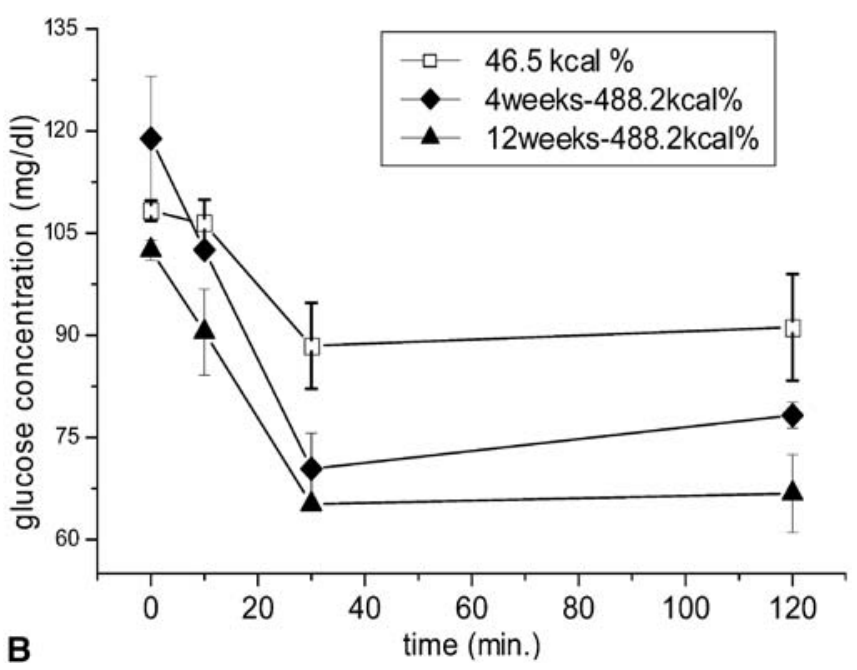

Fig. 2A, B. Effects of high-fat diet on insulin tolerance. Insulin tolerance tests (ITT) were performed in overnight-fasted animals as described in methods. (A) Circulating insulin after ITT in hamsters fed control (46.5 kcal \% as fat) and hypercaloric diet (488.2 kcal \% as fat) for 4 and 12 weeks. (B) Circulating glucose in response to insulin injection in the same groups as in (A). At 10 min the 12-week group was statistically different ( $p=0.037 \mathrm{vs}$ control); at $30 \mathrm{~min}$ and $120 \mathrm{~min}$ time point both high-fat groups were significantly different from controls (30 $\min p=0.027$ and 0.019 respectively) $120 \mathrm{~min}$ the $p=0.034$ and 0.018 respectively)

hepatic glucose output (HGO) and to stimulate glucose disposal.

Further confirmation of this, came from the $\mathrm{R}_{\text {HOMA }}$ (as a measure of insulin tolerance) that gave mean similar values whether the animals were fed a regular (control) or high-fat diet. Together these data indicate that in hamsters, feeding them with a high-fat diet does not cause peripheral insulin resistance.

Effects of high-fat diets on insulin secretion. The absence of insulin resistance suggested a defect in insulin secretion. Therefore, in this experiment we measured circulating insulin concentrations after glucose injec- 


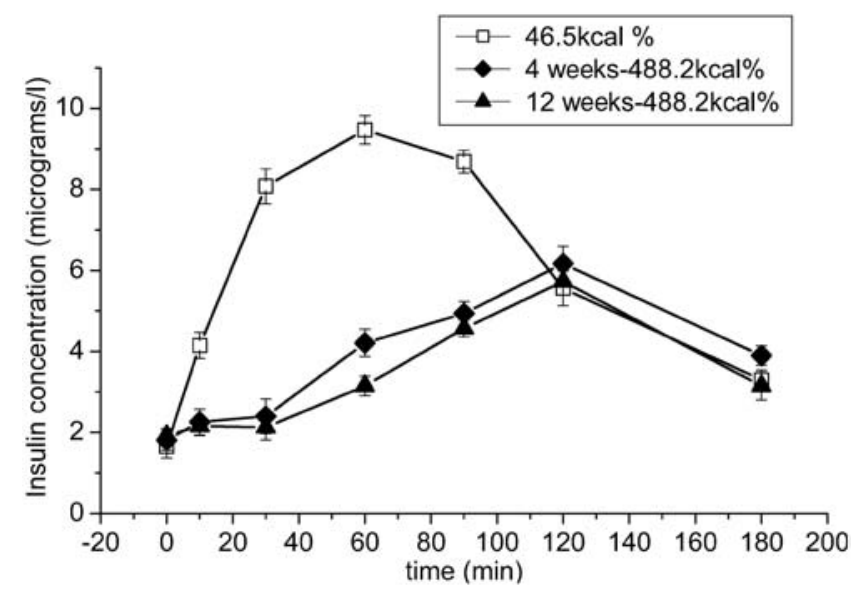

Fig. 3. Effects of high-fat diet on glucose stimulated insulin secretion. Male hamsters fed the $488.2 \mathrm{kcal} \%$ diet for 4 and 12 weeks were fasted for 18 hours prior to i.p. injection of $1 \mathrm{mg}$ glucose (in saline)/g body . Blood was withdrawn from the retro-orbital plexus at indicated times, and insulin concentrations were determined as described in Material and Methods

tion at different time points, i.e. at 4, 12 and 20 weeks of hypercaloric feeding. The results showed that the amount of insulin secreted in response to insulin was greatly reduced, and the timing of the insulin secretion was greatly delayed in animals fed a high-fat diet when compared to animals fed the control diet (Fig. 3).

Effects of high-fat diets on the structure of the pancreas. Electron micrographs of the pancreatic tissue showed that the high-fat diets caused structural alteration of the acinar and beta cells and the effect was most prominent with the $488.2 \mathrm{kcal} \%$ fat diet. At 1 week under the latter diet, we observed sparse lysosome-like structures within the pancreatic acinar cells; after 4 weeks collagenous fibrosis was focally apparent, whereas at 20 weeks extended areas of fibrosis and frequent autophagic vacuoles were noticed (Fig. 4). The structure of the beta cells was not significantly modified during the first 2 weeks of feeding $488.2 \mathrm{kcal} \%$ as fat. Starting with 4 weeks of the diet, beta cells in various stages of degranulation were observed and the amount of granules with less electronopaque content/beta cells increased in time up to about $40 \%$ at 10 weeks, and about $60 \%$ at 20 weeks (Fig. 5A). The capillaries within the endocrine pancreas were affected soon after the initiation of the hypercaloric diet. Thus after 1 week damage of the pericytes was observed; at 2 weeks, two layers of capillary basement membrane were apparent in some locations, and the thickness of the extracellular matrix was about $0.266 \mu \mathrm{m}$, approximately a 1.77 -fold increased above the normal. At 4 weeks of the diet, the thickness of the extracellular matrix was about $0.42 \mu \mathrm{m}$, i.e. 2.8 -fold augmented compared to normal. At 12 weeks the capillary endothelial cells showed a multilayered basal lamina (thickness $\sim 0.70 \mu \mathrm{m}, \sim 4.6$-fold increased above normal) and in numerous locations the enlargement of the pericapillary space was observed facilitating the formation of edema (Fig. 5A). These structural alterations are in contrast to the normal pancreatic capillaries that showed a thin endothelial basal lamina and a narrowed pericapillary space (mean thickness $\sim 0.150 \mu \mathrm{m}$ ) (Fig. 5B).

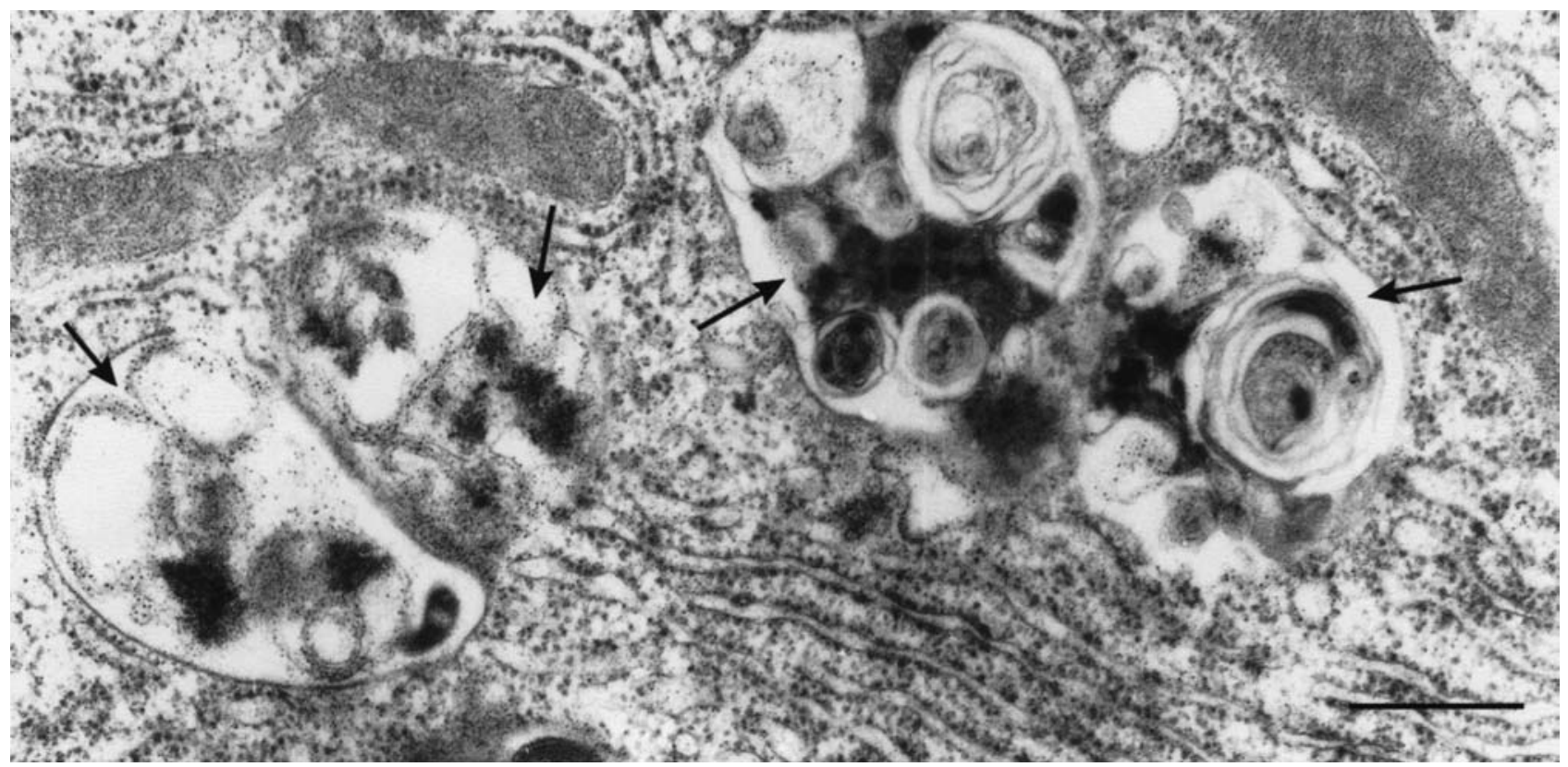

Fig. 4. Effect of high-fat diet on the structure of the acinar pancreatic cell. After 20 weeks of feeding the hamsters with a diet containing $488.2 \mathrm{kcal} \%$ as fat, the pancreatic acinar cells showed frequent autophagic vacuoles (arrow), a structural alteration that may impede their normal secretory potential. Bar $1 \mu \mathrm{m}$ 


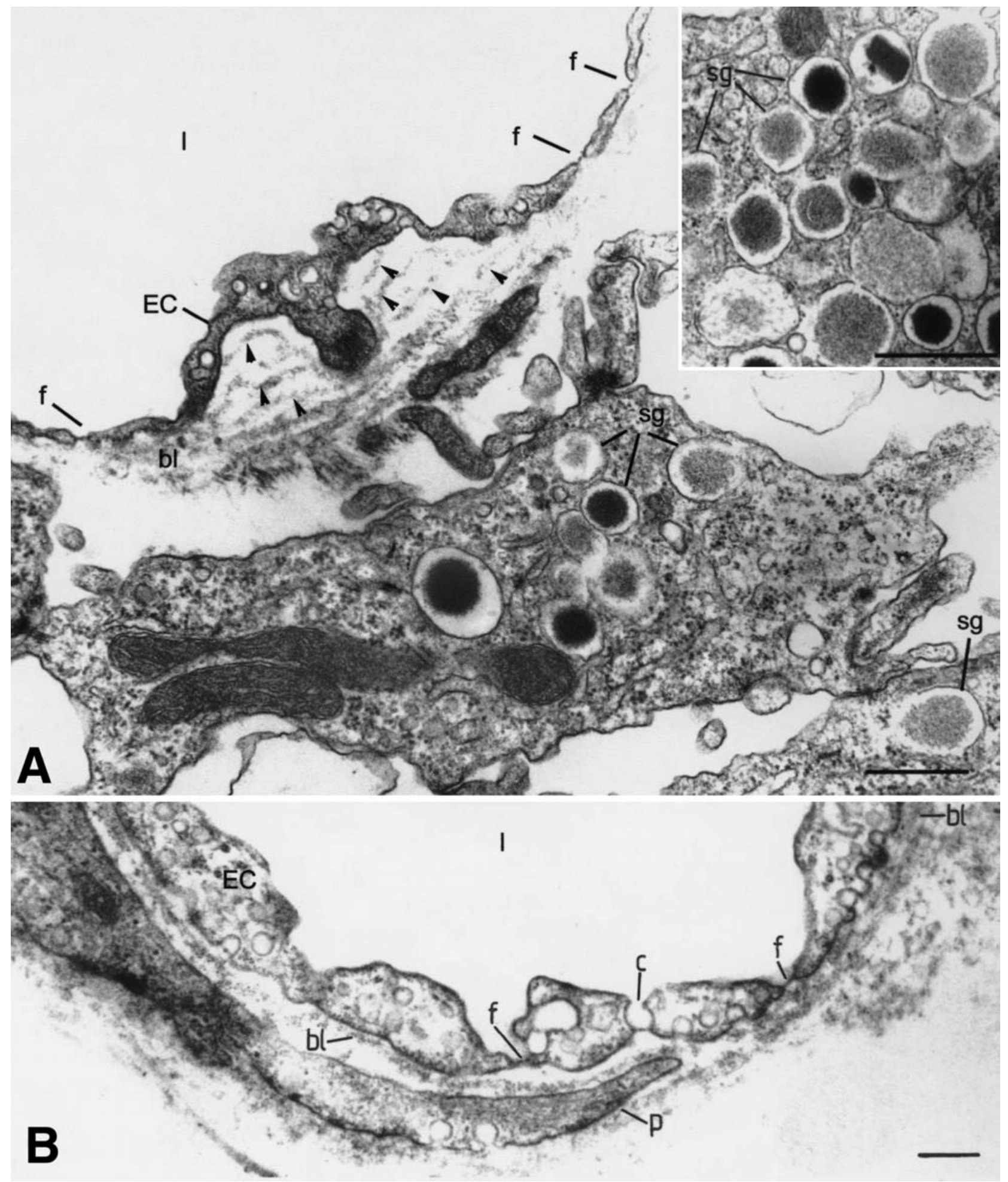

Fig. 5A, B. Effects of high-fat diet on the structure of the endocrine pancreas. A A representative electronmicrograph of a capillary adjacent to a $\beta$-cell in the endocrine pancreas of a hamster fed saturated fat diet $(488.2 \mathrm{kcal} \%$ as fat $)$ for 12 weeks. Note the multilayered (arrow heads) basal lamina (bl) that separates the capillary from the adjoining $\beta$-cell, and the enlargement of the pericapillary space, suggesting interstitial oedema. The $\beta$-cells display secretory granules (sg) full of electrondense contents and numerous granules with less electrondense product, indicative for various stages of degranulation (inset) and impairment of insulin stores. B The structure of a pancreatic capillary in a control hamster (fed $46.5 \mathrm{kcal} \%$ as fat). Note the thin, single layer of basal lamina (bl), and the integrity of the structure of the pericyte $(p) . l$ capillary lumen, $E C$ endothelial cell, $f$ fenestrae, $c$ channel. Bars: a: $0.55 \mu \mathrm{m}$; inset: $0.76 \mu \mathrm{m}$; b: $0.27 \mu \mathrm{m}$ 

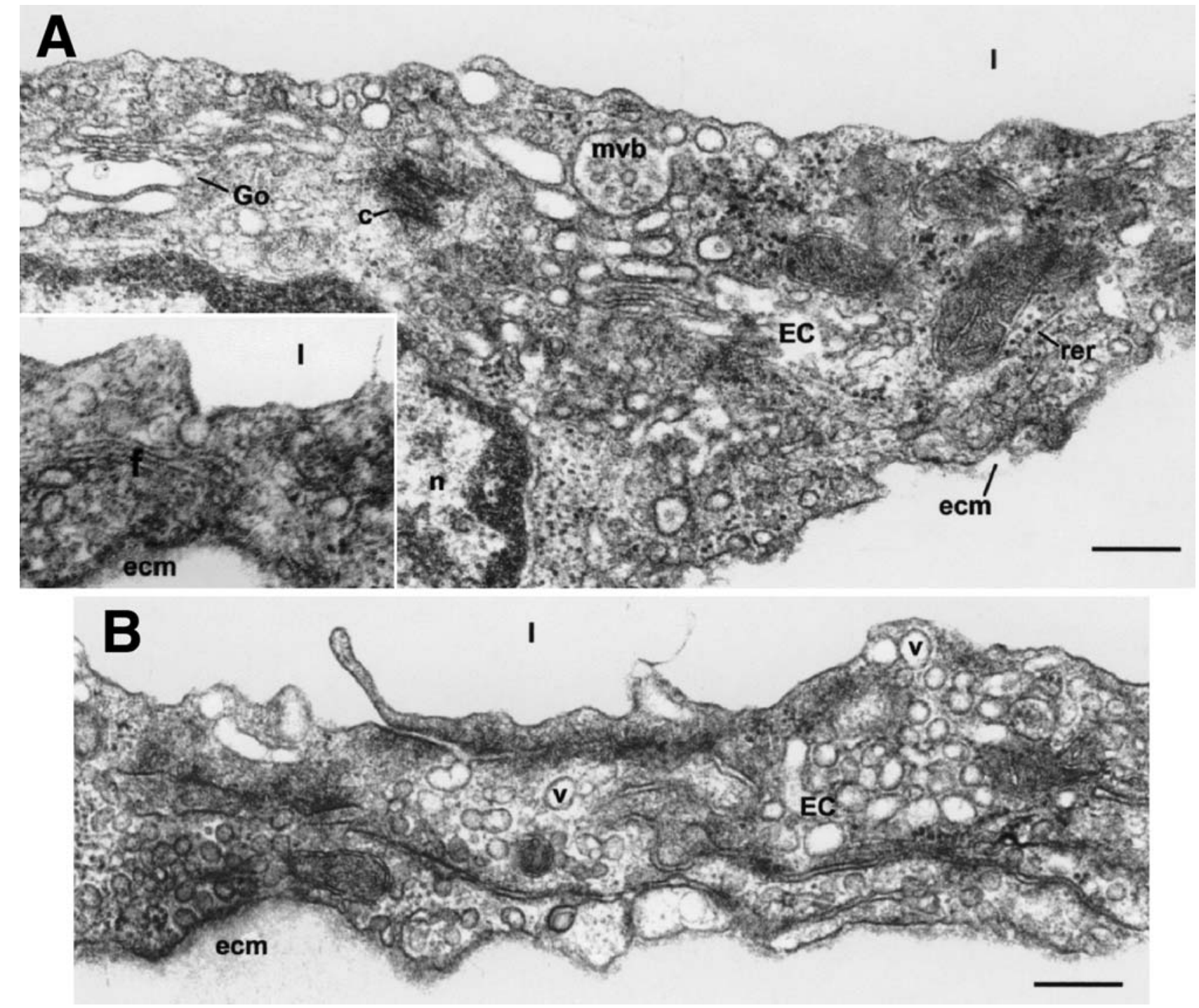

Fig. 6A, B. The structure of the aortic endothelium. A Representative electron micrographs of the structure of the endothelium in the aorta of hamsters fed $488.2 \mathrm{kcal} \%$ as fat for 10 weeks. Note the active metabolic phenotype of the endothelial cell (EC) endowed with abundant Golgi cisternae (Go), rough endoplasmic reticulum (rer) and degradative compartments, such as the multivesicular body (mvb). Inset: a zone containing numerous microfilaments (f). B The aortic endothelium of a hamster fed normal diet ( $46.5 \mathrm{kcal} \%$ as fat). Note the normal phenotype of the cell that contains plasmalemmal vesicles (v) involved in transport processes. $l$ vascular lumen, $c$ centriole, ecm extracellular matrix. Bars: $0.27 \mu \mathrm{m}$

Effect of high-fat diet on the structure of the aortic wall. In animals fed with a high-fat diet, the structure of endothelial cells covering the aortic arch showed evidence of an active metabolic phenotype expressed by abundant rough endoplasmic reticulum and Golgi complex, the presence of multivesicular bodies, and a highly developed cytoskeleton (Fig. 6A). Within the intima some of the smooth muscle cells showed the aspect of necrotic cells. In hamsters fed $46.5 \mathrm{kcal} \%$ as fat, the aortic endothelium showed a normal phenotype. The synthetic and degradative organelles were of rare occurrence, and the cytoplasm contained numerous plasmalemmal vesicles commonly involved in transport processes (Fig. 6B).

Effect of high-fat diet on the structure of the glomerular capillaries. The glomerular capillaries in hamsters fed $46.5 \mathrm{kcal} \%$ had a thin basement membrane $(\mathrm{GBM})$ of $115 \pm 4 \mathrm{~nm}$ (Fig. 7A). The GBM was modified in time by feeding the high-fat diets. The thickness of the GBM gradually increased under the diets made of 267.3 and $488.2 \mathrm{kcal} \%$ as fat, starting from 2 weeks and up to 20 weeks reaching mean values of $258 \pm 12 \mathrm{~nm}$ and $439 \pm 16 \mathrm{~nm}$, respectively. In addition, after 4 weeks of high-fat diet the thickened GBM focally developed enlargements and electron-dense nodules that apparently compress and diminish the vascular capillary lumen (Fig. 7A). At 8 to 10 weeks of diet, prominent multiple layers of GBM were observed within the nodular structures (Fig. 7B); the latter became more elaborated and frequent after 10 to 20 

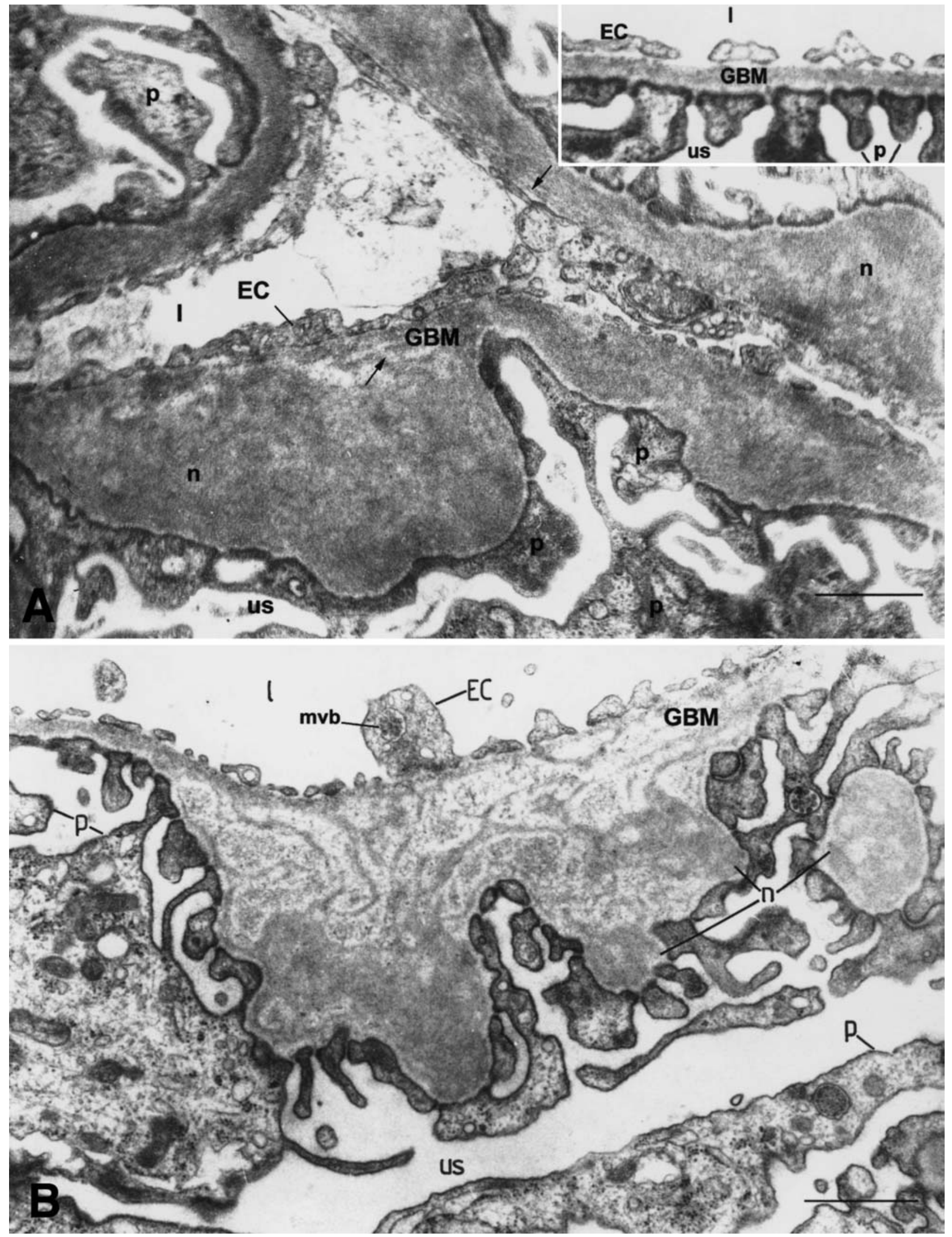

Fig. 7A, B. Effect of high-fat diet on the ultrastructure of the kidney glomerular capillaries. A Hamster fed saturated fat diet (488.2 kcal \% as fat) for 4 weeks. Note the large increase in the thickness of GBM, the formation of intercalated nodular structures $(n)$ and the initiation of the development of a multilayered GBM (arrow); inset: Hamster fed normal diet (46.5 kcal \% as fat). Note the homogeneous, single-layer
GBM. B After 20 weeks of feeding the saturated fat diet, the endothelial cells of glomerular capillaries display a highly reticulated, multilayered GBM with intercalated elaborated nodules in several locations, and a diminished urinary space (us). $l$ vascular lumen, $E C$ endothelial cell, $p$ podocyte, $m v b$ multivesicular bodies. Bars: a and inset: $0.76 \mu \mathrm{m}$; b: $1 \mu \mathrm{m}$ 
weeks and contributed to the diminishment of the urinary space (Fig. 7B). In addition, from early time points, some of the podocytes had lost the slit diaphragms and after 20 weeks of feeding the hamsters with the high-fat diets, the mesangial area was increased about 3.2-fold above the size in control hamsters.

\section{Discussion}

In this study Golden Syrian hamsters were fed diets high in saturated fat and the following modifications were observed: (i) the animals became moderately diabetic as a result of a defect in insulin secretion, rather than by peripheral insulin resistance; (ii) the animals did not become obese, but suffered from a range of severe structural alterations not previously described in the high-fat fed rat and mouse models such as the multilayered basal lamina in the pancreatic capillaries (possibly explaining the delay in insulin secretion), the change in the phenotype of endothelium of the aortic arch (that could impede normal blood vessel function), and the severe modifications of kidney glomeruli, suggesting that the diets high in saturated fat could be an independent risk factor for nephritis.

The high-fat diet (saturated fat) caused a number of perturbances of homeostasis in hamsters including a progressive increase in fasting plasma glucose concentrations accompanied by a progressive decrease in glucose tolerance. However, a surprising feature of the model was that during hypercaloric diets the hamsters lost weight, which was associated with a loss of visceral adipose tissue. We assume weight loss in our animals is related not only to a decreased food intake, but also to the development of other pathologies, such as those in the kidney. However, this differs from humans and other rodent models where visceral fat accumulation would be expected after the consumption of a high-fat diet [32, 33]. However, in rodents the susceptibility to the development of obesity seems to be highly strain specific $[4,34]$. For example, consumption of a high-fat diet by Osborne-Mendel rats led to obesity [11], whereas the rat strains that are not prone to obesity show only a moderate increase in body weight when fed a high-fat diet [7].

Another surprising feature was that while glucose tolerance was progressively impaired in the hamsters, they did not develop insulin resistance and did not show increased postprandial insulin concentrations. This indicates that the insulin is appropriately stimulating glucose uptake into peripheral tissues, an increase which could be linked to the loss of fat, and a related decrease in adipokines, such as TNF- $\alpha$, that would be expected. Defects in hepatic glucose output (HGO) can also contribute to defects in glucose tolerance in animals on a high-fat diet [35]. However, the insulin tolerance tests indicate that insulin is probably shutting down hepatic glucose production in these animals although we cannot rule out a defect in basal HGO contributing to the increase in fasting plasma glucose.

Our results are in contrast with another study [15]. They reported decreased glucose tolerance combined with high serum insulin concentrations in high-fat fed hamsters, although they did not undertake insulin tolerance test. Their diet contained fructose and their animals became obese so it is possible that the insulin resistance was secondary to these factors.

The effects of fat feeding on glucose metabolism in humans is not absolutely clear mostly because of the difficulty of running human dietary intervention studies. The studies that have been carried out generally find that high-fat feeding causes an impairment of glucose tolerance $[1,2,3]$, but in one study where insulin sensitivity was carefully measured by clamping studies it was found that insulin sensitivity actually increased [36].

That the high-fat diet results in both a delay and a decrease in total insulin released into the blood after a glucose load is of interest because defects in insulin secretion kinetics are also a feature of glucose-intolerant humans [37]. Although high-fat diets can induce beta-cell apoptosis and a progressive decline in insulin content in rodents [38], we did not find any major loss of beta cells or any signs of inflammation mediated by the immune system in the pancreas of high-fat fed hamsters. However, the beta cells presented an apparent reduction in the number of dense core granules indicating deficient insulin storage. Furthermore, the significant thickening of the basal lamina surrounding the capillaries in the pancreas suggest that the rate of influx of glucose from the blood stream and efflux of insulin to the blood stream are both likely to be delayed. The possibility that similar defects could contribute to delay insulin secretion in humans has not been studied.

One of our aims was to investigate how high-fat diet enriched in saturated fatty acids alter the plasma lipid profile. Feeding the hamsters with saturated fat caused hypertriglyceridaemia which probably results from a combination of factors including the reduced adipose tissue mass as high concentrations of triglycerides were reported to occur also in the circulation of the hamsters that have been subjected to lipectomy and that were fed high-fat diets [14].

The aortic arch of the hamsters fed with high-fatdiets showed modification of the endothelial cells towards a secretory phenotype and alteration of the structure of smooth muscle cells, that both could be symptoms of modified vascular reactivity that could provide a substrate for the later development of atherosclerotic lesions.

One of the major pathologies caused by the highfat feeding was the gradual alteration of the normal function of the kidney, as evidenced by increasing 
concentrations of creatinine. In the hamsters fed with saturated fat diets the glomerular filtration barrier was found severely modified by thickening and formation of nodules (nodular glomerulosclerosis) that contributed to the narrowing of both vascular and urinary spaces. These modifications can induce an increased glomerular capillary pressure and cause a perturbed renal haemodynamic function. Concurrently, the widening of the mesangial area occurred, and led eventually to renal failure, the main cause of increased mortality recorded in hamsters fed with saturated-fat diets. Our data corroborate and extend the findings of previous studies showing that obesity and high-fat diets are linked with kidney dysfunction in rats $[6,8]$ and dogs [12]. The exact mechanism of this has not been defined but could involve input from non-esterified fatty acids [39]. However, plasma triglycerides have also been implicated as a predictor of renal failure in Type 2 diabetes [37].

The hypertrophy of the mesangium (one of the features of diabetic nephropathy) was also observed in Goto Kakizaki rat, a model of non-insulin-dependent diabetes mellitus [41]. A range of mechanisms have been implicated in the alterations in the structure of the kidney including the diacylglycerol-protein kinase C-extracellular regulated kinase pathway [42] and TGF-beta. Overexpression of TGF-beta was observed in kidneys of dogs fed a high-fat diet [12]. However, in these previous studies it was always assumed that the defects in the kidney were secondary to insulin resistance, obesity or frank diabetes but our results suggest that in the conditions of high-fat feeding they occur even when relatively mild diabetes is present and in the absence of insulin resistance or obesity. This suggests that the high saturated fat diet can be an independent risk factor for the development of nephritis.

Taken together our results show that hamsters fed a diet high in saturated fat developed perturbed homeostasis associated with mild diabetes caused by defects in insulin secretion rather than by insulin resistance or obesity. This was also coupled with severe structural alterations of pancreatic acinar and beta cells, of the vascular wall, and glomerular capillaries. These observations suggest that the hamster fed with saturated-fat diet would be a new model for studying the pathogenic features of high-fat intake, and for separating the effects of the dietary lipids from other confounding factors such as insulin resistance and obesity.

Acknowledgements. This work was supported by the Romanian Academy, the Diabetes UK, the Short Term Scientific Mission Grants from the COST B17 Action "Insulin resistance, obesity and diabetes mellitus in the elderly" of the EU, the Wellcome Trust, and the Royal Society, UK. The authors are indebted for the dedicated work of M. Isachi who helped us monitor the animal model during the experiment.

\section{References}

1. Storlein LH, Baur LA, Kriketos AD et al. (1996) Dietary fats and insulin action. Diabetologia 39:621-631

2. Lichtenstein AH, Schwab US (2000) Relationship of dietary fat to glucose metabolism. Atherosclerosis 150:227243

3. Hu FB, Dam RM van, Liu S (2001) Diet and the risk of Type-II diabetes. Diabetologia 44:805-817

4. Scrocchi LA, Drucker DJ (1998) Effects of aging and a high fat diet on body weight and glucose tolerance in glucagon-like peptide-1 receptor $^{-/}$mice. Endocrinology 139: 3127-3132

5. Dithmar S, Sharara NA, Curcio CA et al. (2001) Murine high-fat diet and laser photochemical model of basal deposits in Bruch membrane. Arch Ophthalmol 119:1643-1649

6. Matsuda S, Arai T, Iwata K, Oka M, Nagase M (1999) A high-fat diet aggravates tubulointerstitial but not glomerular lesions in obese Zucker rats. Kidney Int 56:S150-S152

7. Peiser C, McGregor GP, Lang RE (2000) Leptin receptor expression and suppressor of cytokine signaling transcript levels in high-fat-fed rats. Life Sci 67:2971-2981

8. Jayapalan S, Hossein Saboorian M, Edmunds JW, Aukema HM (2000) High dietary fat intake increases renal cyst disease progression in han: SPRD-cy rats. J Nutr 130:23562360

9. Libal-Weksler Y, Gotlibovitz O, Stark AH, Madar Z (2001) Diet and diabetic state modify glycogen synthase activity and expression in rat hepatocytes. $J$ Nutr Biochem 12:458-464

10. Asai A, Miyazawa T (2001) Dietary curcuminoids prevent high-fat diet-induced lipid accumulation in rat liver and epididymal adipose tissue. J Nutr 131:2932-2935

11. Fitzgerald SM, Henegar JR, Brands MW, Henegar LK, Hall JE (2001) Cardiovascular and renal responses to a high-fat diet in Osborne-Mendel rats. Am J Physiol Regul Integr Comp Physiol 281:DXLVII-DLII

12. Henegar JR, Bigler SA, Henegar LK, Tyagi SC, Hall JE (2001) Functional and structural changes in the kidney in the early stages of obesity. J Am Soc Nephrol 12:12111217

13. Hiramatsu S, Grill V (2001) Influence of a high-fat diet during chronic hyperglycemia on $\beta$-cell function in pancreatic islet transplants to streptozotocin-diabetic rats. Eur J Endocinol 144:521-527

14. Weber RV, Buckley MC, Fried SK, Kral JG (2000) Subcutaneous lipectomy causes a metabolic syndrome in hamsters. Am J Physiol Regul Integr Comp Physiol 279:R936R943

15. Wang PR, Guo Q, Ippolito M et al. (2001) High fat fed hamster, a unique animal model for treatment of diabetic dyslipidemia with peroxisome proliferator activated receptor alpha selective agonists. Eur J Pharmacol 427:285-293

16. Schrauwen P, Wagenmakers AJ, Marken Lichtenbelt WD van, Saris WH, Westerterp KR (2000) Increase in fat oxidation on a high-fat diet is accompanied by an increase in triglyceride-derived fatty acid oxidation. Diabetes 49:640646

17. Dwyer TM, Banks SA, Alonso Galicia M et al. (2000) Distribution of renal medullary hyaluronan in lean and obese rabbits. Kidney Int 58:721-729

18. Bressler P, Bailey SR, Matsuda M, DeFronzo RA (1996) Insulin resistance and coronary heart disease. Diabetologia 39:1345-1350

19. Yip J, Facchini FS, Reaven GM (1998) Resistance to insulin-mediated glucose disposal as a predictor of cardiovascular disease. J Clin Endocrinol Metab 83:2773-2776 
20. DeFronzo RA, Ferrannini E (1991) Insulin resistance: A multifactorial syndrome responsible for NIDDM, obesity, hypertension, dyslipidemia and atherosclerotic cardiovascular disease. Diabetes Care 14:173-194

21. Laakso M (1999) Hyperglycemia and cardiovascular disease in type-2 diabetes. Diabetes 48:937-942

22. Ohtani H (1990) Effects of dietary cholesterol and fatty acids on plasma cholesterol and hepatic lipoprotein metabolism. J Lipid Res 31:1413-1422

23. Sullivan MP, Cerda JJ, Robbins FL, Burgin CW, Beatty RJ (1993) The gerbil, hamster and guinea pig as rodent models for hyperlipidemia. Lab Anim Sci 43:575-578

24. Nistor A, Bulla A, Filip DA, Radu A (1987) The hyperlipidemic hamster as a model of experimental atherosclerosis. Atherosclerosis 68:159-173

25. Simionescu M, Popov D, Sima A et al. (1996) Pathobiochemistry of combined diabetes and atherosclerosis studied on a novel animal model: the hyperlipemic hyperglycemic hamster. Am J Pathol 148:997-1014

26. Popov D, Simionescu M (1997) Alterations of lung structure in experimental diabetes, and diabetes associated with hyperlipidemia. Eur Respir J 10:1850-1858

27. Mc Pherson CW (1987) Selective normative data for the Syrian Golden hamster. In: Van Hoosier GL Jr, McPherson CW (eds) Laboratory hamsters. Academic Press, Harcourt Brace Jovanovich, Orlando Austin San Diego New York Boston London Sydney Tokyo Toronto, p 301

28. Emoto M, Nishizawa Y, Maekawa K et al. (1999) Homeostasis model assessment as a clinical index of insulin resistance in type 2 diabetic patients treated with sulfonylureas. Diabetes Care 22:818-822

29. Raji A, Williams GH, Jeunemaitre $X$ et al. (2001) Insulin resistance in hypertensives: effect of salt sensitivity, renin status and sodium intake. J Hypertens 19:99-105

30. Radziuk J (2000) Insulin sensitivity and its measurement: structural commonalities among the methods. J Clin Endocrinol Metab 85:4426-4433

31. Williamson JR, Rowold E, Hoffman P, Kilo C (1976) Influence of fixation and morphometric techniques on capillary basement membrane thickening. Prevalence data in diabetes. Diabetes 25:604-613
32. Doucet E, St-Pierre S, Almeras N et al. (2002) Reduction of visceral adipose tissue during weight loss, Eur J Clin Nutr 56:297-304

33. Seidel JC, Bouchard C (1997) Visceral fat in relation to health: is it a major culprit or simply an innocent bystander? Int J Obes 21:626-631

34. Surwit RS, Petro AE, Parekh P, Collins S (1997). Low plasma leptin in response to dietary fat in diabetes- and obesity-prone mice. Diabetes 46:1516-1520

35. Kraegen EW, Clark PW, Jenkins AB, Daley EA Chisholm DJ, Storlien LH (1991) Development of muscle insulin resistance after liver insulin resistance in high fat fed rat. Diabetes 40:1397-1403

36. Parillo M, Rivellese AA, Ciardullo AV, Capaldo B, Giacco A, Genovese S, Riccardi G (1992) A high monounsaturated fat/low carbohydrate diet improves peripheral insulin sensitivity in non-insulin dependent diabetic subjects. Metabolism 41:1373-1378

37. Pratley RE, Weyer C (2001) The role of impaired early insulin secretion in the pathogenesis of Type II diabetes mellitus. Diabetologia 44:929-945

38. Donath MY, Gross DJ, Cerasi E, Kaiser N (1999) Hyperglycemia-induced $\beta$-cell apoptosis in pancreatic islets of Psammomys obesus during development of diabetes. Diabetes $48: 738-744$

39. Thomas ME, Harris KPG, Walls J, Furness PN, Brunskill NJ (2002) Fatty acids exacerbate tubulointerstitial injury in protein overload proteinuria. Am J Physiol Renal Physiol 283:F640-F647

40. Colhoun HM, Lee ET, Bennett PH et al. (2001) Risk factors for renal failure: the WHO multinational study of vascular disease in diabetes. Diabetologia 44:S46-S53

41. Phillips AO, Baboolal K, Riley S et al. (2001) Association of prolonged hyperglycemia with glomerular hypertrophy and renal basement membrane thickening in the Goto Kakizaki Model of Non-Insulin-Dependent Diabetes Mellitus. Am J Kidney Dis 37:400-410

42. Haneda M, Koya D, Kikkawa R (2001) Cellular mechanisms in the development and progression of diabetic nephropathy: activation of the DAG-PKC-ERK pathway. Am J Kidney Dis 38:S178-S181 